\title{
Las estrategias discursivas de los artesanos huicholes en el marco turístico*
}

\author{
ROZENN LE MUR**
}

\begin{abstract}
Discursive Strategies of Huicholes Craftsmen Within A TOURIST FramewORK. The sale of Huichol/Wixárika craftwork to tourists has rapidly increased in the last decades. Now it appears to be one of the main tourist attractions in Western Mexico. This paper explains how Huichol craftsmen and artists respond to the tourist gaze in a strategic way, to accomplish economic, butalso political goals. Based on fieldwork and the analysis of in-depth interviews with Huichol artists, it is examined how they continuously create and re-create their culture in dialogue with other social groups.
\end{abstract}

Key words: cultural encounter, touristart, folklorization, narratives, tourist imaginary

\begin{abstract}
Resumen
La venta de artesanía y de arte huichol/wixárika en el circuito turístico se desarrolló muy rápidamente en las últimas décadas. Ahora se le presenta como uno de los mayores atractivos del occidente de México para el turismo. En este artículo, se verá de qué forma los artesanos y artistas huicholes responden a la mirada del turista de manera estratégica, para cumplir con propósitos económicos, pero también políticos. A partir del trabajo de campo realizado y del análisis de entrevistas a profundidad a artesanos huicholes, se examina cómo éstos crean y recrean continuamente su cultura en diálogo con otros grupos sociales.
\end{abstract}

Palabras clave: encuentro cultural, arte turístico, folclorización, narrativas, imaginario turístico

\section{Introducción}

$\mathrm{L}$ a cultura huichola, o wixárika en su propio idioma, con frecuencia es presentada en las páginas web de agencias de viajes como uno de los principales atractivos turísticos de México, sobre todo en la promoción de paquetes turísticos en la región de Jalisco y Nayarit. Su cosmogonía y su artesanía se promueven como un elemento central del patrimonio mexicano. Su iconografía, junto con sus asociaciones de colores vivos, por ser tan fácilmente identificables, hace que los huicholes sean uno de los grupos indígenas mexicanos más reconocibles tanto en el nivel nacional como internacional. Asimismo, existen cada vez más paquetes turísticos centrados en ellos: "Los huicholes, cultura indemne a través del tiempo", "Ruta huichol”, "Experiencia huichol", "Huichol indian village tours", "Encuentro huichol", son algunos de los viajes que se pueden contratar en diferentes agencias.

Los huicholes constituyen uno de los cuatro grupos indígenas que viven en la región conocida como el Gran Nayar, en la porción meridional de la Sierra Madre Occidental en México. Su territorio tradicional se extiende

*Artículo recibido el 04/12/17 y aceptado el 20/02/18.

** Universidad de Guadalajara, Departamento de Estudios de la Comunicación Social. Av. José Parres Arias núm. 150, San José del Bajío, 45132, Zapopan, Jalisco, México <r_lemur@hotmail.com>. 
sobre cuatro estados: Jalisco, Nayarit, Durango y Zacatecas. En los años setenta, con los programas gubernamentales Huicot y Coplamar ${ }^{1}$ se pusieron en marcha políticas indigenistas masivas que afectaron profundamente a las comunidades huicholas de Jalisco y Nayarit. La comercialización de la artesanía huichola fue uno de los objetivos mayores de estos programas, e influyó mucho el hecho de que su venta se transformara en una de las estrategias de sobrevivencia de mayor trascendencia para este grupo. Aunado a ello, el interés de los antropólogos y comerciantes hacia esta artesanía hizo que su mercado se expandiera a gran velocidad. Algunas tiendas con este giro empezaron a trabajar como talleres donde se formaron los primeros artesanos huicholes reconocidos como artistas.

El desarrollo de la artesanía huichola en los años sesenta coincidió claramente con el aumento del interés del turismo internacional por esta cultura, en particular por parte de la comunidad new age, fascinada por lo que reconocía como una cultura espiritual y psicodélica. Desde esa época, y de manera creciente hasta la fecha, estas condiciones de apertura al turismo nacional e internacional hacen que los huicholes manejen su identidad de una nueva forma. Son lo que Phil Weigand (1992: 16) llama indios profesionales, que viven de su folclore. Para ganarse el sustento, ponen en evidencia lo más interesante, impactante y exótico de su cultura. Moldean la percepción del pasado y de las tradiciones de las comunidades indígenas, transforman los mitos para que adopten un "formato" más atractivo para los turistas. Estos procesos se pueden relacionar con lo que Eric Hobsbawm y Terence Ranger (1983) describieron como invención de la tradición y Edward Said (1980) delimitó como geografía imaginada.

Los artesanos y artistas huicholes tienen conciencia de lo que atrae a los turistas a visitarlos, y de lo que John Urry (2002) designó tourist gaze. Asimismo, la "mirada" no se dirige sólo en un sentido, sino que turistas y artistas huicholes coinciden en una situación dialógica, un mutual gaze (Maoz, 2006). Se trata de encontrarse con el imaginario de los visitantes, de alimentar el interés en el otro, y de desconectar al turista de su ambiente conocido. Estos procesos de adaptación al turismo se denominan estrategias discursivas, que pueden ser respuestas tanto de resistencia como de apropiación, que Gabriela Coronado y Bob Hodge
(2004: 69) describieron como un "tipo de negociación intercultural en la que los receptores responden activamente en la construcción de nuevos significados”.

Las narrativas del imaginario alrededor de la cultura huichola se construyen desde varias fuentes. Los actores sociales participantes en el circuito turístico tienen diferentes objetivos, prioridades y métodos. Es importante notar que hoy en día, además de ser un sustento económico para los huicholes, el comercio de su cosmogonía y artesanía también constituye un negocio extremadamente rentable para otros agentes: comerciantes mestizos, inversionistas extranjeros, agencias de viajes locales, etcétera. No se debe olvidar que los conflictos existentes entre instituciones, tiendas, agencias, no son sólo ideológicos, sino que tienen una repercusión financiera considerable. Por lo tanto, hay tipos de intereses y opiniones muy variados en cuanto a la adaptación de los huicholes al turismo, así como distintas visiones sobre su papel, las ideas de vulnerabilidad, autonomía, pérdida cultural, etcétera.

En este trabajo nos centraremos en el análisis de las estrategias discursivas de los artesanos y artistas huicholes en este ambiente y nos preguntaremos de qué forma responden a estos variados discursos. ¿Cómo crean y recrean continuamente su cultura, se adaptan a múltiples tipos de presiones y hacen suyos elementos externos de manera estratégica?

Nuestro análisis se basará en el trabajo de campo realizado de 2010 a 2013 en las zonas turísticas en las cuales los artesanos y artistas venden sus piezas (sobre todo en Jalisco y Nayarit). La comunidad huichola de San Andrés Cohamiata o "Tateikie", en la Sierra Madre Occidental en el norte de Jalisco, desarrolló un programa de ecoturismo, y recibe cientos de turistas nacionales y extranjeros en Semana Santa, por lo cual efectuamos trabajo de campo en esta zona. Sin embargo, la mayor parte de él la llevamos a cabo en lugares de contacto con el turismo nacional y extranjero, en particular en las ciudades de Puerto Vallarta, Guadalajara y Tepic. El trabajo de campo se dividió en dos aspectos: la descripción general del "circuito turístico" (entrevistas con comerciantes de arte y artesanía huicholes, curadores de museos, agentes de viajes que proponen "huichol tours", y encuestas a turistas nacionales y extranjeros) y ocho entrevistas a profundidad efectuadas a artesanos y artistas huicholes de la región. ${ }^{2}$ Los criterios de selección de su perfil fueron

1 El programa Huicot (centrado en la región Huicot, síntesis de las palabras huichol, cora, tepehúan, las tres comunidades indígenas que habitan esta zona) fue implementado de 1970 a 1976, y el Coplamar (Coordinación General del Plan Nacional de Zonas Deprimidas y Grupos Marginados), de 1976 a 1982.

2 Antonio López Pinedo, Francisco Bautista, Justo Benítez, Antonio Mejía, Juan Carrillo, Iginio Bautista, Maurilio Rentería y Cilau Valadez. 
a) que tuvieran características similares (en particular la venta de sus piezas en el ambiente turístico) y b) que divergieran en otros rasgos: lugar de residencia, edad, comunidad de origen y sitio de venta. Junto con los artistas y artesanos huicholes, según los preceptos de metodología horizontal (Corona y Kaltmeier, 2012), reflexionamos sobre cómo ha evolucionado la elaboración de la artesanía huichola y sobre las estrategias económicas y políticas que aplican en este contexto. ${ }^{3}$

Nuestro enfoque no será el de descalificar los procesos de adaptación al turismo como pérdida de la cultura, o como falsedad, sino examinarlos en cuanto procesos de comunicación y encuentro cultural. Mostraremos cómo los huicholes urbanos, en especial los jóvenes, crean y recrean continuamente su cultura, se adaptan a distintas clases de presiones y hacen suyos elementos externos de forma estratégica.

A continuación, describiremos estas estrategias siguiendo tres ejes: el desarrollo de la educación y el aprendizaje de herramientas discursivas, los procesos de divulgación y asociación estratégica con diferentes grupos, y la comercialización de la etnicidad.

\section{Estrategias de educación y aprendizaje de herramientas discursivas}

\section{La educación, factor decisivo de valorización de la cultura}

El tema de la educación de los jóvenes indígenas en México no es sencillo. ¿Cómo aplicar un sistema que permita ofrecerles las mismas oportunidades que a cualquier otro ciudadano mexicano sin que pierdan en el proceso el lazo con sus comunidades de origen y su cultura?

Como señalan Séverine Durin y Angélica Rojas (2005: 149):

entre los wixaritari serranos, la escuela suele ser vista como un "mal necesario" ya que promete enseñar a los niños a hablar, escribir y leer en español. A su vez es concebida como una actividad que contribuye a alejar a los niños de su cultura, pues esta institución ajena compite con la socialización tradicionalmente a cargo de los mayores.

Denis Lemaistre (2004) también describe el sistema escolar como una fuente de conflictos y contradicciones para los jóvenes huicholes. Opina que la mayoría de los alumnos indígenas sale del sistema educativo en una posición frustrante. Según él, la escuela les causa dudas sobre el valor de su costumbre sin ni siquiera darles, en contraparte, la capacidad de adquirir un trabajo bien remunerado ni un lugar influyente dentro de su comunidad.

Además, Guillermo de la Peña (1995) subraya la discriminación hacia los niños indígenas en el medio escolar, en las ciudades, e incluso de parte de los profesores.

Sin minimizar los conflictos que acabamos de indicar, lo que queremos mostrar en esta sección es que la mayoría de los jóvenes huicholes entrevistados, educados en la ciudad y que ahora viven de la venta de su artesanía o arte, mencionan a la escuela como un factor decisivo y muy valioso respecto a su aceptación en la ciudad en cuanto indígenas. La definen como el elemento esencial en el reconocimiento de su cultura, tanto por parte de la sociedad mayoritaria como de ellos mismos.

Iginio Bautista, quien dirigió por unos años su propia tienda de arte huichol en el centro de Puerto Vallarta, comenta que cuando empezó a asistir a la secundaria en Tepic iba vestido "de mestizo, y con una mochila”. Le daba vergüenza usar su traje y verse como huichol. Lo explica diciendo que "no sabía": "allí fue donde aprendí que todos somos iguales, no más con diferentes culturas. Allí aprendí también que todo lo que hacemos, con esto podía salir adelante. $O$ también allí aprendí que no me diera vergüenza andar así, con mi traje”.

Los artistas y artesanos entrevistados concuerdan en que las iniciativas de algunos maestros de organizar actividades durante las cuales exponían su trabajo de artesanos frente a todos los alumnos, respondiendo a sus preguntas y vestidos con su traje tradicional, fueron claves en cuanto a la valoración de su cultura. A partir de esos momentos, se apropiaron de su identidad étnica de manera estratégica.

No hay duda de que esta visión optimista del papel de la escuela en la valorización de su cultura está basada en historias de vida exitosas de huicholes artesanos; es muy probable que otros huicholes hayan tenido en la escuela experiencias menos gratificantes. Sin embargo, el testimonio de estos casos exitosos parece indicar que el hecho de que los huicholes hagan presentaciones sobre su cultura en los salones de clase, o que se relacionen con otros grupos indígenas en la escuela, son factores positivos para su reconocimiento por la sociedad, pero sobre todo entre sí.

3 Trabajos previos al presente estudio son Le Mur (2015a y 2015b). 
Si se piensa la interculturalidad de un modo en que ésta sobrepase la simple convivencia y no implique una discriminación sistematizada, queda claro que el ámbito educativo es crucial. Las actividades propuestas respecto a la diversidad cultural y la valoración de la interculturalidad en la escuela son elementos decisivos en cuanto al sentimiento de aceptación de los jóvenes huicholes y a la forma en la cual éstos se relacionan con otros grupos.

\section{El aprendizaje de herramientas discursivas}

Muchos padres de familia huicholes escogen migrar a Guadalajara o a Tepic o enviar a sus hijos a estos centros urbanos para que vayan a la escuela. La razón principal por la cual deciden que deben educarse en la ciudad es para que dominen el idioma español. Como las autoridades wixaritari expusieron en una carta al presidente de la República el 8 de septiembre de 1996:

Las palabras sirven para aclarar o para confundir, las palabras se utilizan también para dominar. El que maneje bien las palabras puede mandar a quien no maneja bien las palabras. El pueblo Wixarika habla su idioma propio que es una lengua muy antigua de México y por medio de ella le cantamos a nuestros dioses para que llueva y haya vida y salud para todos. Por no conocer bien el idioma español hemos sufrido. Nos han considerado ignorantes, analfabetas. Nos han quitado tierras. Han venido a estudiarnos y a decidir por nosotros nuestro destino. Con distintas ideas han venido a nuestra casa a implantar maneras de ver la vida que atentan directamente con nuestra cultura [cit. en Durin y Rojas, 2005: 170].

Los huicholes tienen conciencia de que el dominio del español no es sólo un medio de comunicación, sino que realmente constituye una herramienta discursiva y, como tal, un elemento de poder. Según se puede observar en esta carta, el dominio del español va más allá del conocimiento de las reglas gramaticales o del vocabulario; consiste también en una adaptación a los códigos culturales de la sociedad mayoritaria, para interactuar con ella de manera más favorable.

Antonio Mijares, joven huichol estudiante del Instituto Tecnológico y de Estudios Superiores de Occidente (ITEso) en Ciencias Políticas y portavoz de la asociación de la Wixáritari Artistas y Artesanos Unidos (WAAU), considera que su "doble visión" le permite "entrelazar" a los huicholes con la sociedad mayoritaria. Piensa que, como mediador, tiene la responsabilidad de ayudar al proceso necesario de comunicación entre huicholes y mestizos. Su posición le permite usar géneros discursivos diferentes. Puede dirigirse a los huicholes serranos durante reuniones en sus comunidades, en el idioma wixárika, en su propio contexto. A la vez, tiene la capacidad de hablar con huicholes urbanos, con sus compañeros del ITEso, con la prensa, etcétera, y sorprenderlos utilizando los mismos códigos que ellos. Lo precisa como una estrategia muy eficaz para llamar la atención, salir del marco en el cual sabe que lo definen, lo que al mismo tiempo le es útil para ejercer cierta presión en el nivel institucional y se generen proyectos que incluyan a las comunidades huicholas.

\section{Divulgación y asociación estratégica con diferentes grupos}

\section{El uso estratégico de los medios de comunicación}

Educarse otorga a los jóvenes huicholes herramientas discursivas que también pueden ser tecnológicas. Oscar Ukeme Bautista cuenta que cuando llegó a la ciudad en 1996, lo que más le llamó la atención fue su primer contacto con una computadora. Dice que esta experiencia le dejó claro que a través de internet y desde cualquier parte del mundo se podía dar a conocer todo, y de ahí supo que quería estudiar informática para él también poder hacerlo.

En años recientes, la utilización de los medios de comunicación ha sido decisiva en cuanto a la reivindicación de objetivos políticos y al reconocimiento de la cultura huichola. Se creó, por ejemplo, el Frente en Defensa de Wirikuta, Tamatsima Wa Haa, formado por las autoridades tradicionales, civiles y agrarias de las comunidades wixáritari de Jalisco, Nayarit y Durango y coordinado por la Asociación Jalisciense de Apoyo a los Grupos Indígenas (AJAGI). En su página web $^{4}$ explica por qué la actividad minera en Real de Catorce es una agresión a uno de los lugares sagrados más importantes para los huicholes. Esta página es frecuentemente actualizada con diversos documentos: artículos publicados en diferentes periódicos y distintos tipos de videos, como el cortometraje Entre lo profano y lo divino (2012), realizado por Rubén Albarrán, el vocalista del grupo Café Tacuba, y por el cineasta Ángel Flores.

\footnotetext{
4 http://frenteendefensadewirikuta.org.
} 
Hacerse conocer en todo el mundo se ha vuelto un objetivo para muchos huicholes. Algunos aprenden inglés para poder comunicarse con más personas. Los sitios web tienen secciones traducidas a este idioma. Realizan exposiciones en varios lugares de México y en el extranjero. Por ejemplo, los artistas huicholes Francisco Bautista y Cilau Valadez pasan mucho tiempo fuera del país: participan en ferias, exposiciones o entrevistas en Estados Unidos, Canadá o Europa. Asimismo, el uso de Facebook tuvo un gran crecimiento en los últimos cinco años: ahora, además de ser un espacio de expresión personal para muchos jóvenes huicholes, es una herramienta con la cual articulan venta de piezas artesanales o artísticas y propósitos políticos.

A principios de la segunda década del siglo xxi, sólo existían unas cuantas páginas web donde se podía comprar artesanía y arte huichol, y todas, sin excepción, estaban manejadas por mestizos o extranjeros. Hoy en día, hay muchas más plataformas de este estilo, y la proporción de artesanos huicholes que venden sus piezas en línea por su cuenta, presentándose como ellos quieran, también ha aumentado drásticamente.

Para los huicholes, la divulgación de su cultura, de sus proyectos y de su artesanía o arte se ha vuelto una estrategia económica y política muy eficiente. Los artesanos y artistas huicholes en la ciudad participan a menudo en entrevistas filmadas, en conferencias de prensa, e incluso en congresos, por ejemplo en la Universidad de Guadalajara o en la Universidad Nacional Autónoma de México.

\section{La unión estratégica de los huicholes entre sí y con otros grupos}

En numerosas ocasiones, el discurso oficial del gobierno celebra el pluralismo cultural, mientras deja de lado las cuestiones económicas y sociopolíticas fundamentales. Representa a los pueblos indígenas como una de las mayores riquezas de México, usa sus costumbres y artesanías como atractivo turístico, firma acuerdos legales para defender los derechos indígenas en el nivel cultural, de protección de sitios sagrados, etcétera, sin que los involucrados sean consultados de manera oficial.

Muchos artesanos huicholes se están organizando ahora para hacerle frente a esta situación. Varias asociaciones están trabajando en conjunto para cambiar la dinámica de su relación con el gobierno. Como Maurilio Rentería, artista huichol y director de la WAAU, quien explica que su misión es levantar la voz, llamar la atención internacional sobre el desinterés del gobier- no, la mercantilización de su imagen sin su permiso, el desprecio de sus lugares sagrados. En el proceso quieren también obtener beneficios inmediatos, por ejemplo, espacios dignos para la venta de artesanía y arte o créditos para empezar sus negocios; sin embargo, la meta real, de largo plazo, es la reconsideración del proyecto nacional para que sea verdaderamente pluricultural.

Una de las estrategias que aplican los huicholes es aliarse como grupo unido, sobrepasando las diferencias de opinión que puedan tener, en particular entre las diversas comunidades. Tal vez desde un punto de vista exterior esta posición parezca bastante natural, ya que se difunde (y en especial en el contexto turístico) una imagen del pueblo huichol como sociedad unida, sin conflictos internos. La realidad es muy diferente, pues existen desacuerdos entre las distintas comunidades tradicionales, ligadas sobre todo a la conservación de las tradiciones. Por ejemplo, las autoridades de la comunidad de Santa Catarina Cuexcomatitlán no aprueban la apertura al turismo de San Andrés Cohamiata.

Los huicholes no sólo se unen entre sí; se alían asimismo con otros grupos, lo que ha resultado una de sus estrategias más eficientes para divulgar su cultura y cumplir con sus objetivos económicos y políticos. En los últimos años, realizaron muchas actividades

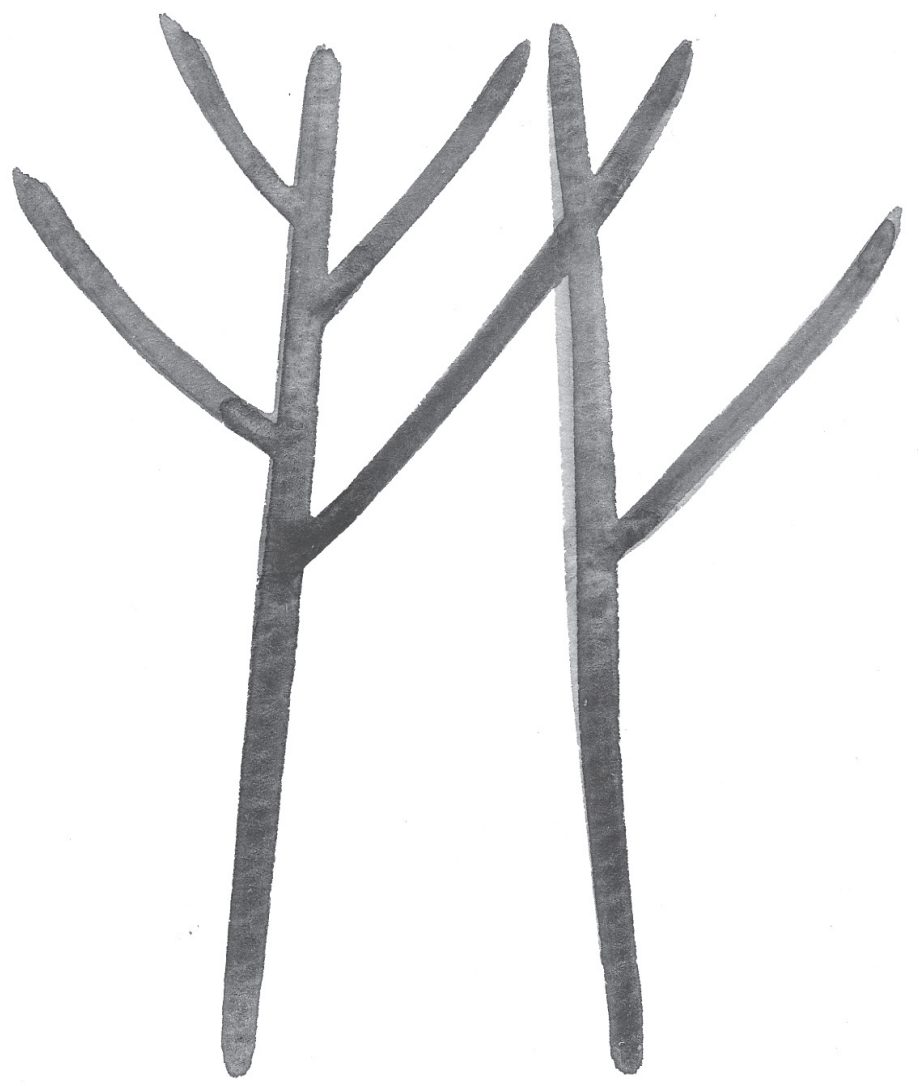


de todo tipo para abrirse y convivir con otros grupos, sobre todo con los jóvenes. Se destacan cuatro con los cuales se han asociado estratégicamente: académicos, ecologistas, artistas y diseñadores gráficos. Existen cada vez más productos modernos diseñados con la integración de la iconografía huichola, así como aplicaciones y cortometrajes de animación. Se pueden mencionar las aplicaciones diseñadas por el Centro Cultural de España en México "Vamos a aprender mixteco" o "Vamos a aprender náhuatl", o los cuentos indígenas animados narrados en su lengua originaria 68 voces, creados por Gabriela Badillo y COMBO, en los cuales se retratan de manera moderna las leyendas de diferentes pueblos indígenas.

Estas asociaciones estratégicas son fundamentales para que, desde la niñez, los huicholes urbanos puedan valorar su condición de intermediarios, vinculando la riqueza de su cultura con los elementos de la sociedad mayoritaria. Además, enseña a los niños mestizos de México a integrar componentes de las culturas indígenas a su cotidianidad, lo que podría dar pie a una generación de ciudadanos mexicanos que se relaciona de forma distinta con los pueblos indígenas.

\section{Estrategias de comercialización y marketing de la etnicidad}

\section{La comercialización de la artesanía $y$ arte huicholes como motor de creatividad}

Más que muchas otras industrias, el turismo tiene la capacidad de transformar culturas. Estas transformaciones casi siempre son vistas, en particular en el medio académico, como una consecuencia desastrosa de la democratización del turismo. Con frecuencia se alude al "turismo irresponsable", "descontrolado" e "ignorante", que altera socialmente a las comunidades anfitrionas.

La preocupación central, desde esta perspectiva, radica en la cuestión de la pérdida cultural. Se argumenta que los elementos más importantes de las culturas se transforman en acontecimientos turísticos, y al ser representados sistemáticamente de este modo "espectacularizado" y "descontextualizado", pierden poco a poco sus significados simbólicos y rituales. Se describe la producción de imágenes prototípicas y simplificadoras de las sociedades, y donde la vida de toda una población queda resumida en algunas escenas, bailes u objetos comercializables. La descripción de estos procesos, definidos como folclorización, se puede encontrar, por ejemplo, en los trabajos de Pazos (1998) y Grillot (2001).
Varios antropólogos especializados en el estudio de las comunidades huicholas y de la evolución de su artesanía defienden puntos de vista similares. Muestran que los procesos de adaptación a la demanda turística hacen que la artesanía que se vende o las danzas que se bailan para los turistas se alejan mucho de las culturas a las cuales dicen reproducir. Subrayan una simplificación de la cultura en el ámbito turístico, sobre todo en la elaboración de la artesanía. Señalan que se reproducen ciertos modelos y se copian las figuras centrales, variando sólo los colores.

Acelia García de Weigand (2002: 154) nota que un morral huichol que en principio tenía 18 flores de peyote se redujo a cuatro, y es probable que los diseños más bellos se copien en procesos industriales y se produzcan en masa, igual que los sarapes navajo. Según ella, el resultado será una decadencia de los textiles producidos por los huicholes, aun cuando serán más numerosos que nunca.

En el mismo tenor, Olivia Kindl (2003) hace comentarios en cuanto a la fabricación de las jícaras. Apunta que, como los otros objetos destinados por los huicholes a la venta, éstas se hallan sujetas a cambios estilísticos o temáticos que dependen de la demanda comercial. Indica que la presión económica adquiere en este caso una importancia decisiva, en detrimento de la calidad técnica, el valor estético y la dimensión cultural del objeto elaborado.

Juan Negrín (1977) opina que la venta masiva de artesanías en la ciudad, o en el circuito turístico, generó una simplificación de los motivos y una pérdida de la calidad de las piezas de artesanía. En lugar de la "verdadera actividad creadora", se reproducen de manera sistemática las mismas formas. De acuerdo con él, algunos huicholes prefieren vender a los turistas una versión acultural de sus artesanías, "sin compromiso", manteniendo a propósito al lado los ritos religiosos que podrían dar otra dimensión a la fabricación de artesanías, justamente para no mezclar lo turístico de mala calidad y la verdadera confección de artesanías. Además, plantea que la producción de artesanía kitsch conlleva una imagen estereotipada que los huicholes proyectan de su cultura hacia el exterior de sus comunidades, una "pseudoidentidad" que hace que se pierda lo acumulado históricamente.

Otros autores enfocados en la artesanía huichola, como Beatriz Bastarrica Mora (2004), Jorge Arturo Chamorro Escalante (2007) y Sarah Corona Berkin (2002), centraron más bien su análisis en el aspecto comunicacional y contextual de su evolución. Demuestran que las críticas a la pérdida cultural de las artesanías huicholas suelen ser de índole esencialista, y a menudo no concuerdan con la opinión de los propios artesanos 
y artistas huicholes. Como subraya Corona Berkin (2002: 24), "la religión huichola es tradicionalmente más abstracta que figurativa: sus kaliwei, o lugares de adoración, no están decorados y sólo algunas veces se encuentran dentro objetos votivos temporales, como flechas o cuernos de venado". Las piezas de artesanía muy coloridas apenas se desarrollaron en los años sesenta, lo que pone en perspectiva cualquier debate histórico sobre su evolución.

Asimismo, esta evolución reciente de la artesanía huichola ligada al turismo entraña varios aspectos que considerar. Por supuesto, existen artesanías huicholas con diferentes niveles de calidad. La adaptación al turismo es más o menos sutil, muchos artesanos huicholes fabrican exactamente lo que les piden las tiendas o empresas con las cuales trabajan, y la calidad de los resultados es variada, involucrando más o menos la decisión de cada artesano. En las tiendas de artesanías huicholas ubicadas en el malecón de Puerto Vallarta, las esculturas de madera forradas de chaquira son producidas en serie; cualquiera puede encontrar allí todos los posibles animales que podrían faltar en su colección personal, desde el oso polar hasta los hipopótamos, y si no los hallan en el color o el tamaño deseados, los artesanos que trabajan en estas tiendas se los elaboran tal como quieren.

Varios artesanos nos explicaron que su prioridad es que los productos sean vendibles y competitivos (pues muchos no reciben un sueldo y sólo les pagan cuando venden alguna pieza), y que emplean menos tiempo en su realización.

Sin embargo, es importante precisar dos puntos: primero, tienen muy clara la distinción entre la artesanía que elaboran para la venta y aquella que realizan en otros contextos (con sus hijos, o que servirán de ofrenda). Además, no consideran que las piezas producidas en las tiendas sean de mala calidad, o que no representen dignamente a la cultura huichola. Antonio Mejía, artesano huichol quien vende sus piezas en un tianguis de Guadalajara, plantea que ven esto "como una manera de ganarse la vida y que sirve de primer acercamiento a la cultura huichola para muchos turistas".

Aunque los productos de artesanía huichola en el contexto turístico puedan llegar a ser estereotipados, y reflejen de modo demasiado obvio los gustos de los turistas, en raras ocasiones la artesanía es "mal hecha" o de "mal gusto". Los ejemplos que se podrían calificar así y con los cuales nos hemos encontrado (cuadros huicholes de las películas Ghost Busters o Star Wars, o el uso de material barato o de colores fluorescentes) no fueron muchos, y en la mayoría de los casos eran piezas pedidas con esas especificaciones y que no fue- ron recogidas por los clientes. En otros casos, ilustran una falta de diálogo con el cliente potencial, ya que no se reconocen como huicholas, y no son la mayoría. La utilización de lo étnico por parte de los huicholes es un proceso que se ha producido a lo largo de la historia y que creció de modo considerable a partir de los años sesenta. Muchos artesanos vieron cómo sus padres e incluso sus abuelos manejaron su etnicidad en el contexto turístico. Observaron cuáles son las piezas más vendidas, cuál fue la evolución de los gustos de los turistas, etcétera. De hecho, la comercialización de la artesanía en el marco turístico, además de ser una fuente de ingresos para muchos huicholes, fue precisamente lo que permitió que se desarrollara la artesanía y el arte de este grupo. Impulsó la elaboración de diferentes tipos de piezas, algunas muy repetidas y otras muy inspiradas.

Además, en el caso de la artesanía huichola, mercantilizar la producción artesanal generó la realización de objetos muy finos. Si no fuera por la comercialización de la artesanía, las piezas que hoy en día son vendidas como arte no existirían: se desarrollaron con y para el turismo.

Ahora, se pueden ver cada vez más ejemplos de artistas huicholes que demuestran que la comercialización de su artesanía y de su imagen en cuanto huicholes puede ser un proceso productivo y creativo. En particular para los más jóvenes, la comercialización de la etnicidad no implica una pérdida cultural; al contrario, permite el renacer y la divulgación de su cultura. Una de las estrategias más decisivas que tratan de aplicar los artesanos huicholes ahora consiste en tomar el control de la comercialización de su artesanía y de la divulgación de su cultura. Idealmente, quisieran poder vender su artesanía en sus propias tiendas y dominar todos los pasos de la comercialización, desde la elección de los materiales y motivos en la elaboración de las piezas hasta el dominio de la publicidad. Para lograrlo, un paso fundamental es el control de su imagen.

\section{La importancia de la narración para la venta, invención de la tradición y manejo de la autenticidad}

El trabajo sobre la narrativa del circuito turístico parece ser uno de los dispositivos más fundamentales de la empresa turística. Desde los primeros estudios sociológicos efectuados sobre el turismo, se pone de relieve la dualidad de los viajes turísticos. Los turistas buscan a la vez encontrarse con lo más auténtico de las culturas locales y considerarse como viajeros y no 
como turistas, realizar de cierta forma un peregrinaje moderno y, al mismo tiempo, cortar con la realidad. Daniel Boorstin (1964) arguye, anticipando el trabajo de Jean Baudrillard sobre la hiperrealidad (1981), que los americanos no pueden experimentar de forma directa la "realidad" sino que pasan por "pseudoeventos". El imaginario simbólico sobre el mundo indígena se construye a partir de elementos disparejos, geográfica y temporalmente. Se mezclan aspectos culturales de indios de todas partes del mundo, junto con diferentes costumbres y creencias religiosas.

La autenticidad se presenta, en particular en el marco turístico, como una característica inherente a los pueblos indígenas. Según McCracken (1988: 106-107), los occidentales sienten que su estilo de vida se vuelve cada vez más inauténtico, entonces imaginan un mundo indígena y auténtico, y lo "consumen". Dentro de este discurso occidental de la autenticidad, lo antiguo es más auténtico que lo actual y lo "hecho a mano" es más auténtico que lo hecho a máquina. Hay personas, textiles, motivos o colores más auténticos que otros.

Este discurso de la autenticidad es una mirada, una construcción imaginada que se forma sobre todo desde el Occidente. Sin embargo, la definición de lo auténtico no siempre responde a los mismos criterios, y puede ser bastante ambigua. Varios investigadores propusieron estudios muy pertinentes en cuanto a la autenticidad en la venta de artesanía y arte en el medio turístico y sus consecuencias sociales (por ejemplo, los trabajos de Dean MacCannell, 1973; Nelson Graburn, 1976, 1999; Bennetta Jules Rosette, 1984; Sally Price, 1989; Chris Rojek y John Urry, 1997; Ruth Phillips y Christopher Steiner, 1999). En tiempos más recientes, autores como Nikki Macleod (2005) siguen reflexionando en torno a las fronteras borrosas y sumamente contextuales en la definición de nociones como la autenticidad.

En el caso de la cultura huichola, muchos elementos que los artesanos y artistas describen como fundamentales para su definición no existían hace unos siglos. No quiere decir que no sean considerados como auténticos o que sean "falsos" elementos de esta cultura. Los cuadros de estambre y las jícaras con mucha chaquira ya son del todo apropiados por los huicholes, aunque hace un siglo no se usaban como ahora.

Pero aun si los huicholes no emplean mucho la noción de autenticidad dentro de su comunidad, o si la utilizan con criterios diferentes, entienden que es un concepto recurrente en el marco turístico. Los artesanos huicholes que desde niños vieron a sus padres vender sus obras en el contexto turístico se dieron cuenta de que esta valoración está muy ligada al entorno en el cual se comercializa la artesanía. El mismo producto no va a ser vendido a igual precio si es presentado en el suelo de un tianguis o en una distinguida tienda en Tlaquepaque.

Mediante el relato, los artesanos construyen para los turistas un imaginario sobre su cultura y su artesanía. Esta narración está hecha para el cliente en función de lo que quiere escuchar, entonces, varios puntos pueden ser inventados o exagerados. Se articula alrededor de lo que Edward Bruner (2005: 4) llama narrativas maestras o metanarrativas, que son las características y estereotipos más llamativos de cada destino turístico. Por ejemplo, las narrativas maestras de Egipto tratan de la época de los faraones, de las tumbas y las pirámides; las del este de África versan sobre los animales y los paisajes salvajes; y aquellas ligadas con los huicholes tienen que ver con el uso del peyote, la autenticidad en su estilo de vida, su conexión con la Madre Tierra y los dioses. Los artesanos huicholes tienen conciencia de la imagen que reflejan en el ámbito turístico, y construyen su propia narrativa para el consumo exterior teniendo en cuenta tales expectativas. Esta construcción puede reforzar o, al contrario, oponerse a las narrativas maestras. Algunas historias inventadas para turistas se están integrando poco a poco a la cultura huichola; muchos turistas están interesados en comprar figuras de mariposas, elefantes, búhos o ranas (respectivamente, "signos de buena suerte", "protectores de la familia", "adivinadores del futuro" y "protectoras de la salud”, según los folletos distribuidos en las tiendas de Puerto Vallarta manejadas por extranjeros).

Lo que buscan los turistas son historias que puedan llevar consigo junto con la pieza comprada; es esencial que sientan que realizan algo más que una simple transacción comercial. De manera estratégica, los artesanos huicholes aprendieron a integrar sus piezas a una narración, y así "esconder" lo comercial en la relación con el turista: escriben el significado de sus motivos detrás de los cuadros de estambre. Esta estrategia, que muy pocos artesanos y artistas aplicaban hace unos años, generó un considerable aumento de la venta de las tablas de estambre. Ahora, prácticamente todos narran la historia de sus cuadros, y, si no logran hacerlo, piden a otros artesanos o a propietarios de tiendas la escriban por ellos.

También, las esculturas rellenas de chaquira son a menudo entregadas al cliente con un folleto descriptivo de su significado, y aunque nunca está redactado por huicholes, ellos se apropian la información que vinculan. Además, los artesanos están a menudo dispuestos a contestar a las preguntas de los turistas. Insisten sobre las narrativas maestras huicholas: ponen 
de relieve el papel del peyote en la elaboración de los cuadros de estambre, explican que la artesanía es una conexión con los dioses, etcétera. Así, se están recreando mitos de origen, rituales olvidados o nuevos emblemas con una gran capacidad de incorporar dentro de un sentido étnico todo tipo de enunciados (Camus, 1997: 166).

\section{Estrategias de adaptación a la demanda Entre modas y costumbres}

Como cualquier negocio, la artesanía huichola se adapta a la demanda de los clientes. Los huicholes tienen conciencia de que su artesanía es un producto y debe ajustarse al cambio en las modas y gustos de los consumidores. Muchos están listos para acceder a las exigencias de sus clientes; por ejemplo, el artista Antonio López Pinedo tiene un catálogo para darles ideas o incluso escoger directamente una pieza. Varios de ellos aceptan reproducir cuadros de estambre a partir de una fotografía del cliente (el retrato de una persona o un animal, paisajes, etcétera). Este tipo de trabajo es lo que en la Casa Huichol ${ }^{5}$ llaman artesanía aplicada (diferenciándolo del arte). Por ejemplo, unos huicholes que se hospedaban en la asociación, hicieron una tabla de surf rellena de hilo de estambre para la tienda Squalo.

En la exposición Wixaritari xatsikayari (La identidad transmitida mediante el relato), se reservó un espacio para los artistas huicholes jóvenes: temari wa nierika (jóvenes creadores). Los cuadros de estambre exhibidos demuestran que la nueva generación de artistas huicholes incluye en ellos una gran cantidad de nuevos elementos. Muchos se unen alrededor del principio de economía solidaria y del comercio justo; estas palabras incluso están incluidas en los cuadros, escritas con hilos de estambre, como en los de Tomasa Montoya González y Silvestre Castro Carrillo. Lucas Matzuwa Castro integra de manera sincrética un símbolo muy fuerte de la mexicanidad, Emiliano Zapata, con la cosmovisión huichola: lo dibuja con una cola de venado (véase figura 1). Se puede ver también la asimilación de elementos cristianos y populares de México en el cuadro Tanana, de Juan Hainiuweme Carrillo Carillo, que plasma a la virgen de Guadalupe con peyotes (véase figura 2).
Figura 1

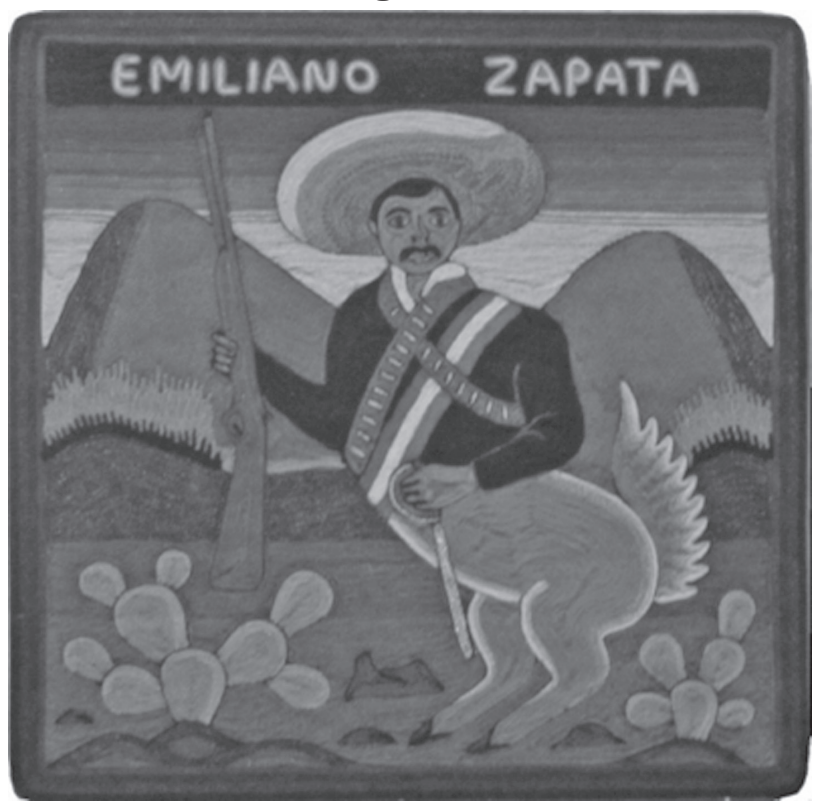

Zapata, de Lucas Matzuwa Castro Jiménez, La Selva Café, Guadalajara, 2012

Figura 2

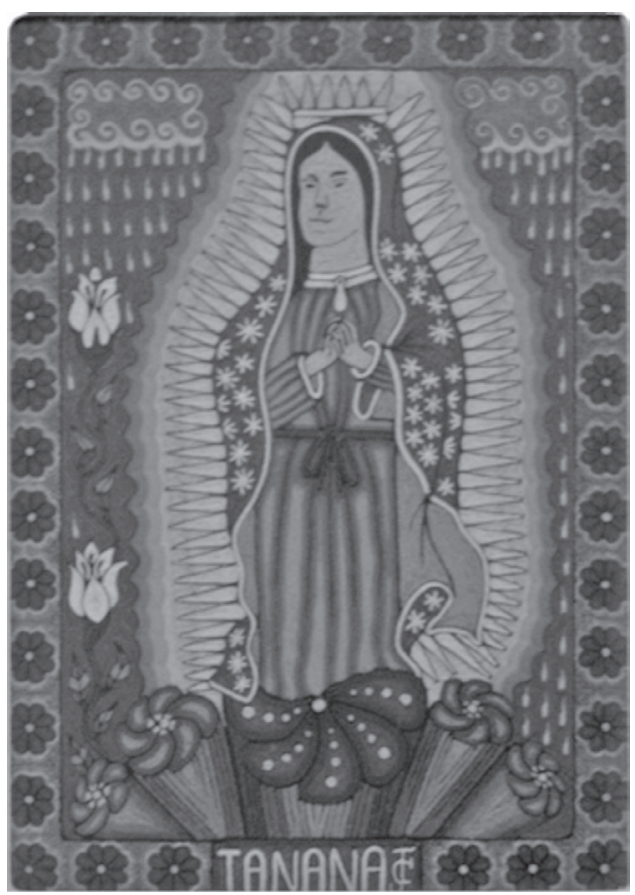

Tanana, de Juan Carrillo, La Selva Café, Guadalajara, 2012

5 Casa Huichol es el proyecto de asistencia de la Asociación para la Investigación, Capacitación y Asistencia Wixárika (AICAw) A. C., en donde se apoya a los enfermos huicholes con asistencia social, capacitación y vínculos para vender su artesanía. 
Esta generación de artistas tiende a señalar que, en las últimas décadas, el arte huichol se ha vuelto un género discursivo fácil de reconocer, por lo cual se permite empujar sus límites, integrar nuevas aportaciones y desarrollar estilos propios. Aun así, los artistas y artesanos se imponen límites en cuanto al sincretismo en sus obras. Gilberto Rivera, quien labora en la Casa Huichol, explica que a veces todos los huicholes hospedados ahí rechazan ciertos tipos de trabajo porque "no son propios". Por ejemplo, un cliente estaba dispuesto a pagar varios miles de pesos para tener un ojo de dios de grandes dimensiones. Ningún artesano quiso realizar esta obra, porque hubiera sido técnicamente difícil de hacer $\mathrm{y}$, sobre todo, porque para ellos no tenía sentido.

En la página web del Frente en Defensa de Wirikuta se especifica que los eventos artísticos y culturales en apoyo a esta causa deben estar "basados en la conciencia y el respeto a la forma de vida de los pueblos indígenas". ${ }^{6}$ No quieren asociarse con empresas, instituciones o ideas contrarias a sus principios: cualquier patrocinio o presencia de marcas comerciales deben ser aprobadas por el SHEWI-Comité de Arte y Cultura del Frente en Defensa de Wirikuta.

En el circuito turístico, estas reglas no son tan estrictas. Empero, algunos elementos de los cuadros de estambre no cambian, para seguir siendo reconocidos como huicholes y no transformarse en mexican curious: siempre son elaborados por huicholes, y deben ser producidos de manera artesanal. La autenticidad del estilo de vida del artesano o artista siempre es central para el cliente o, más precisamente, la comprobación de los criterios de autenticidad del cliente. No se representan aspectos demasiado "modernos" de la sociedad occidental, aunque estén integrados al estilo de vida de los huicholes (coches, televisiones, botellas, etcétera).

Por otro lado, hay aspectos de las artesanías huicholas que evolucionan, en particular en los cuadros de estambre. Se integran dibujos en perspectiva, lo que no se hacía hace unos años. Algunos artistas juegan con el límite entre representaciones figurativas y abstractas. Los márgenes de los cuadros, que tradicionalmente se forman a partir de tres colores de hilos diferentes, se producen ahora de muchos otros modos: con motivos rectangulares, con serpientes, incluso se hacen en relieve o de chaquira. En especial, por el aspecto competitivo de las galerías de arte huichol (que son muy numerosas sobre todo en Puerto Vallarta), ciertos artistas se quieren destacar de modo más radical y proponen piezas novedosas. Desarrollan estilos más personales, y experimentan con nuevos materiales y texturas (véanse figuras 3 y 4).

\section{Figura 3}

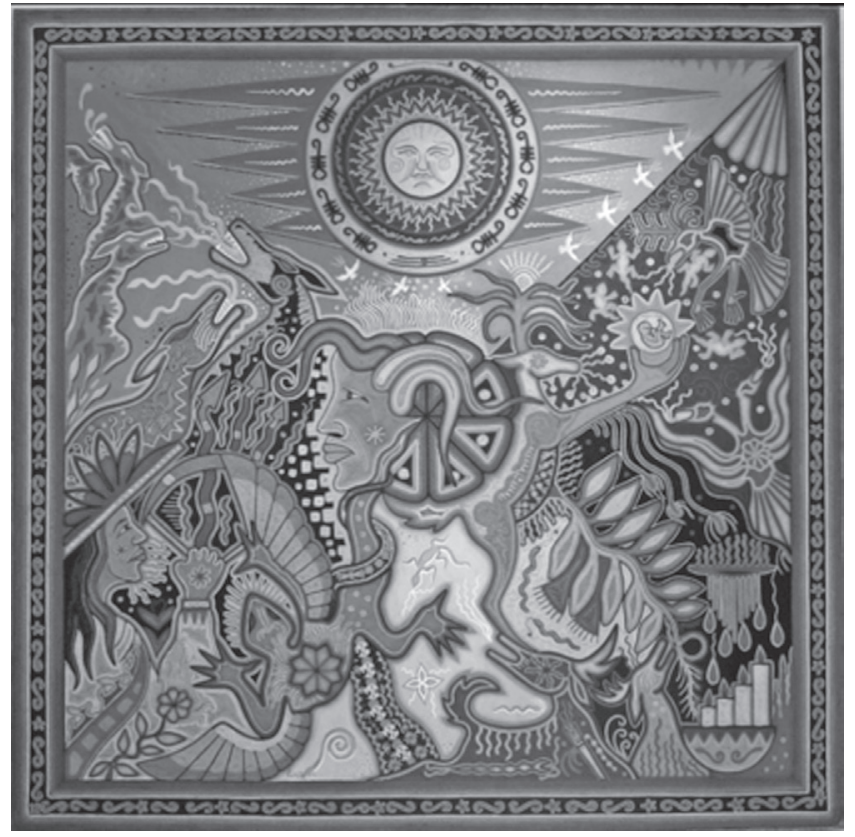

"Sin título", de Neikame, Galería Colectika, Puerto Vallarta, 2011

Figura 4

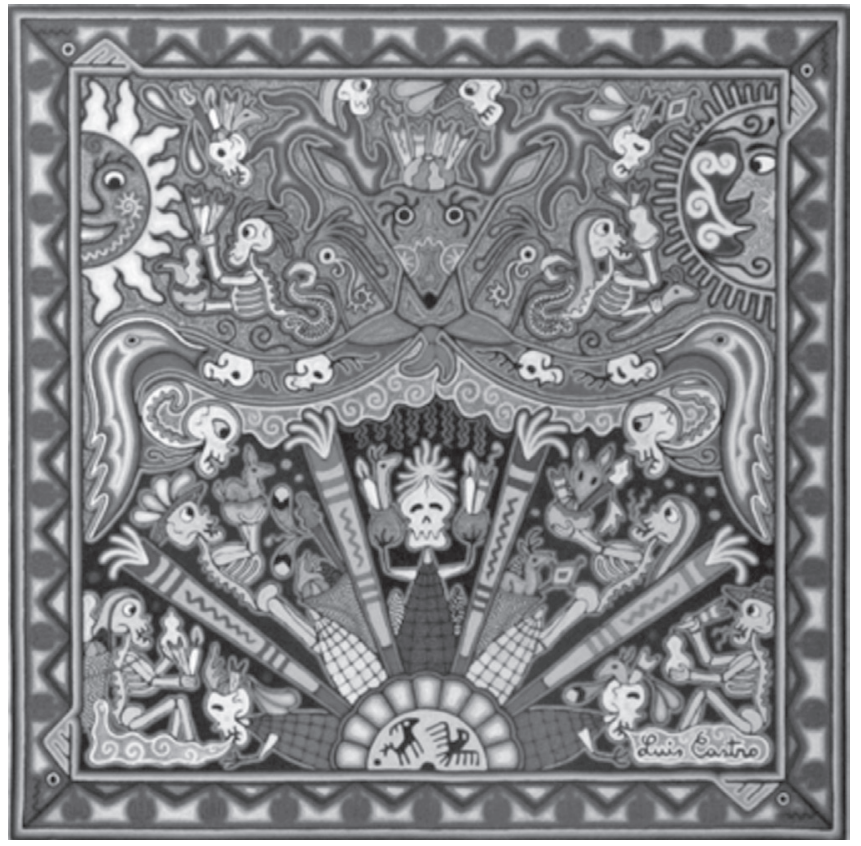

"Sin título", de Luis Casto, Galería Colectika, Puerto Vallarta, 2011

${ }^{6}$ http:// frenteendefensadewirikuta.org. 
No obstante, el contenido temático de los cuadros, aunque varía, debe conservar ciertas connotaciones, que pueden ser, por ejemplo, la espiritualidad, las ceremonias religiosas, el contacto con los dioses, el origen del mundo, la valoración de la naturaleza y la Madre Tierra, entre otras. De hecho, y se puede observar en Puerto Vallarta, es la atracción por los elementos lo más "auténticos" posible; este criterio llega a ser más importante que aspectos de belleza u originalidad. Los clientes quieren comprar objetos con historia, quieren comprar ofrendas (estas piezas son parecidas a lo que David Brown, 1999, llama falsos auténticos).

\section{Conclusiones}

El desarrollo del turismo en Jalisco y Nayarit provocó que los huicholes dedicados a la venta de artesanía reflexionaran sobre su propia identidad y cómo la presentan en el circuito turístico: realizan un arreglo consciente de su discurso.

Vimos que estas estrategias tienen, por supuesto, un objetivo económico: ganarse la vida, pero también político, como lo ponen de manifiesto sus acciones para oponerse a la explotación minera en el sitio sagrado de Wirikuta. De hecho, la visualización política de los huicholes, la venta de su artesanía y arte, el manejo del imaginario en el ámbito turístico ligado a la cosmogonía huichola y la asociación con diferentes grupos son estrategias vinculadas unas con las otras y que sirven a propósitos complementarios.

Las estrategias discursivas aplicadas por la nueva generación de artesanos y artistas huicholes son particularmente interesantes, ya que su posición de intermediarios, el hecho de haber crecido en ambientes diferentes, de conocer a la vez las reglas del mundo occidental y de sus comunidades de origen, les proporciona herramientas discursivas para comunicarse con más éxito con diversos grupos sociales. Logran adaptarse a una clientela sobre todo occidental y, al mismo tiempo, proyectar una imagen de tradición. Proponen artículos que se inscriben en un contexto actual articulándose con las metanarrativas del turismo: la autenticidad, la espiritualidad, la posibilidad de una organización societal distinta, etcétera. Manejan con destreza ajustarse al contexto específico en el cual van a exponer o a vender sus piezas: en tianguis, por internet, en museos, en concursos de artesanías, entre otros.

De esta forma, demuestran que nociones tan centrales en el ámbito turístico como la autenticidad, la pureza cultural o la tradición son conceptos comunicacionales, que se han evidenciado necesarios para conceptualizar al otro, aunque están sujetos a cambios. Las narraciones de los artistas varían en función de los espacios en las cuales se generan: en un contexto turístico, institucional, comunitario, político, etcétera. La tradición y la autenticidad siempre son relevantes y tienen un fuerte peso identitario, pero se interpretan de varias formas.

Aunque queda claro que existen asimetrías de poder en el marco turístico, las estrategias que implementan los artesanos huicholes nos indican que también puede ser un espacio de empoderamiento para ellos. Hay cada vez más ejemplos de huicholes que hacen patente que la venta de la artesanía y la comercialización de su imagen como huichol puede ser un proceso productivo. Para muchos jóvenes, el uso de la etnicidad no implica una pérdida cultural; por el contrario, permite reforzar y divulgar su cultura. Lo que buscan ahora es cambiar las condiciones de mercado, que a menudo no les son favorables. Quieren acreditar que son artesanos profesionales y que producen piezas de calidad, para lo cual piden el establecimiento de reglas justas para vender sus productos.

\section{Fuentes}

Bastarrica Mora, Beatriz

2004 "El simbolismo en el arte de José Benítez Sánchez", tesis de maestría, Universidad de Guadalajara, Guadalajara.

BAUDRILLARD, JEAN 1981 Simulacres et simulations, Galilée, París.

BOORSTIN, DANIEL

1964 The Image: A Guide to Pseudo-events in America, Harper \& Row, Nueva York.

Brown, David 1999 "Des faux authentiques. Tourisme versus

BRUNER, EDWARD pèlerinage”, en Terrain, núm. 33, pp. 41-56.

2005 Culture on Tour: Ethnographies of Travel, University of Chicago Press, Chicago.

Camus, Manuela

1997 "Los profesionistas indígenas y el poder de la piratería cultural”, en Espiral, vol. VII, núm. 8, pp. 147-187

Chamorro Escalante, Jorge Arturo

2007 La cultura expresiva Wixarika. Reflexiones y abstracciones del mundo indígena del norte de Jalisco, Universidad de Guadalajara, Guadalajara.

Corona Berkin, SARAH

2002 Miradas entrevistas, Universidad de Guadalajara, Guadalajara.

Corona Berkin, Sarah y Olaf Kaltmeier

2012 Endiálogo:metodologías horizontales enciencias sociales, Gedisa, Barcelona.

Coronado, Gabriela y Bob Hodge

2004 El hipertexto multicultural en México posmoderno, Centro de Investigaciones y Estudios Superiores en Antropología Social / Miguel Ángel Porrúa, México. 
Durin, Séverine y Angélica RoJas

2005 "El conflicto entre la escuela y la cultura huichola. Traslape y negociación de tiempos", en Relaciones, vol. XXVI, núm. 101, pp. 148-190.

García de Weigand, Acelia

2002 "Las tablas huicholas y su origen", en Phil Weigand (comp.), Estudio histórico y cultural sobre los huicholes, Universidad de Guadalajara, Colotlán, pp. 117-128.

Graburn, NeLson

1976 Ethnic and Tourist Arts. Cultural Expressions from the Fourth World, University of California Press, Berkeley.

Graburn, Nelson

1999 "Ethnic and Tourist Arts Revisited", en Ruth Phillips y Christopher Steiner (eds.), Unpacking Culture. Art and Commodity in Colonial and Postcolonial Worlds, University of California

Grillot, Caroline Press, Berkeley y Los Ángeles, pp. 335-354.

2001 "L'impact de la folklorisation dans l'expression identitaire de la fête de Guzang chez les Miao

HoBsBawm, ERIC de Xijang”, en Ateliers, núm. 24, pp. 69-86.

Y TERENCE RANGER

1983 The Invention of Tradition, Cambridge University Press, Londres.

Jules Rosette, Bennetta

1984 The Messages of Tourist Art: An African Semiotic System in Comparative Perspective, Plenum Press, Nueva York.

KindL, Olivia

2003 La jícara huichola. Estudios monográficos, Instituto Nacional de Antropología e HistoriaConsejo Nacional para la Cultura y las Artes / Universidad de Guadalajara, México.

Le Mur, Rozenn

2015a "La evolución del arte huichol junto al turismo. Entre apreciación y apropiación cultural", en Desacatos, año 15, núm. 49, septiembrediciembre, pp. 114-129.

Le Mur, Rozenn

2015b "Los cuadros de estambre huicholes en el circuito turístico", en Sarah Corona y Rozenn Le Mur (coords.), La negociación para la comunicación intercultural. Los wixáritari ante los desafios actuales, Consejo Nacional para la Cultura y las Artes / Universidad de Guadalajara, México, pp. 172-215.

Lemaistre, Denis

2004 Le chamane et son chant, Recherches et documents, Amériques Latines / L'Harmattan, París.

MacCannell, Dean

1973 "Staged Authenticity: Arrangements of Social
Space in Tourist Settings", en The American Journal of Sociology, vol. 79, núm. 3, pp. 589603.

MACLEOD, NiKKI

2005 "Cultural Tourism: Aspects of Authenticity and Commodification", en Mike Robinson y Melanie Smith (eds.), Cultural Tourism in a Changing World, Channel View Publications, Tonowanda, pp. 177-189.

MAOZ, DARYA

2006 "The Mutual Gaze", en Annals of Tourism Research, vol. 33, núm. 1, pp. 221-239.

McCracken, Grant

1988 CultureandConsumption:NewApproaches to the Symbolic Character of Consumer Goods and Ac-

NEGRÍN, JuAN tivities, Indiana University Press, Bloomington.

1977 El arte contemporáneo de los huicholes, Universidad de Guadalajara/Centro Regional de Occidente/Museo Regional de Guadalajara/ Instituto Nacional de Antropología e Historia/

Pazos, Álvaro Secretaría de Educación Pública, Guadalajara.

1998 "La re-presentación de la cultura. Museos etnográficos y antropología”, en Política y Sociedad, núm. 27, pp. 33-45.

Peña, Guillermo de la

1995 "La ciudadanía étnica y la construcción de los indios en el México contemporáneo", en Revista Internacional de Filosofía Política, núm. 6, pp. 116-140

PhILLIPS, Ruth

y Christopher Steiner

1999 Unpacking Culture. Art and Commodity in Colonial and Postcolonial Worlds, University of California Press, Berkeley y Los Ángeles.

Price, Sally

1989 Primitive Art in Civilized Places, University of Chicago Press, Londres.

ROJEK, CHRIS Y JOHN URRY

1997 Touring Cultures: Transformations of Travel and Theory, Routledge, Londres.

SAID, EDWARD

1980 L'orientalisme. L'orient créé par l'Occident, Le Seuil, París.

URRY, JOHN 2002 The Tourist Gaze, Sage, Londres.

Weigand, PhiL

1992 "Aculturación diferencial entre los indios huicholes", en Phil Weigand, Ensayos sobre el Gran Nayar: entre coras, huicholes y tepehuanos, Instituto Nacional Indigenista/Centro de Estudios Mexicanos y Centroamericanos/El Colegio de Michoacán, México, pp. 153-175. 